\title{
Silicon Photonics Radio-over-Fiber Transmitter using GeSi EAMs for Frequency Up-conversion
}

\author{
K. Van Gasse, J. Verbist, H. Li, G. Torfs, J. Bauwelinck, G. Roelkens
}

\begin{abstract}
In this work we present a silicon photonics Radioover-Fiber transmitter with microwave photonic up-conversion capability. The circuit consists of a pair of parallel GeSi EAMs in a MZI structure. We show that the up-converter/transmitter can up-convert 64-QAM data on a $1.5 \mathrm{GHz}$ IF to any output carrier frequency in the 7-26 GHz range. This interval is only limited by measurement equipment, as we demonstrate that the EAM has a $3 \mathrm{~dB}$ bandwidth exceeding $65 \mathrm{GHz}$. Furthermore, the linearity of the up-converter/transmitter is characterized and a SFDR larger than $82 \mathrm{~dB} \cdot \mathrm{Hz}^{2 / 3}$ is demonstrated. Using this system we transmit up to $1.3 \mathrm{~Gb} / \mathrm{s}$ 64-QAM data over $2 \mathrm{~km}$ of SMF with a received $5.4 \%$ rms EVM.
\end{abstract}

Index Terms-Silicon photonic transceivers, Radio-over-Fiber, Microwave Photonics, Electro-optic mixer

\section{INTRODUCTION}

The development of Integrated Microwave Photonics (IMWP) is a promising technology for the seamless integration of the fiber-optic network and wireless systems such as Radio Access Networks (RANs) and antenna remoting. Silicon photonics has shown to be a very promising technology to implement IMWP systems such as filters [1], integrated microwave-frequency generators [2] and even programmable signal processing systems [3]-[6]. In the next generation mobile network $(5 \mathrm{G})$ the use of carrier frequencies ranging from $6 \mathrm{GHz}$ to $65 \mathrm{GHz}$ is envisioned [7][8], which will require rethinking the RAN architecture. In such 5G RANs, Analogue Radio-over-Fiber (ARoF) could play an important role to enable the ambitious performance goals of 5G. The use of ARoF enables centralized network control and lower deployment cost of small-cell networks. This in turns enables a cost-efficient implementation of cloud-RAN architectures, which are a popular candidate for 5G networks [9]. Another advantage of $\mathrm{ARoF}$, is that the microwave frequency up- or down-conversion stage can be implemented in the optical domain. The use of photonic up-conversion enables wide-band operation and can simplify the transmitter RF-chain. Photonic microwave frequency conversion has been extensively studied in the recent years and has shown promising results [10][11]. This strategy relies on the use of two parallel $\mathrm{LiNbO}_{3} \mathrm{MZMs}$ in a super MZM structure, where one MZM receives the

The authors would like to thank the AFOSR for the grant FA955018-1-0015 Innovative Silicon and InP Integrated Photonic Devices for RF Downconversion"

The authors would like to thank the ERC for the advanced grant 695495 ATTO: A new concept for ultra-high capacity wireless networks"

K. Van Gasse and G. Roelkens are with the Photonics Research Group, Department of Information Technology, Ghent Universityimec, Ghent 9052 , Belgium (e-mail: kasper.vangasse@ugent.be; gunther.roelkens@ugent.be).

J. Verbist, H. Li, G. Torfs and J. Bauwelinck are with IDLab, Department of Information Technology, Ghent University-imec, Ghent 9052, Belgium (email: jochem.verbist@ugent.be. data on a RF carrier and the other is driven with the LO signal. To optimize the conversion efficiency, the super MZM is biased at minimum transmission such that the optical carrier is suppressed. This approach ensures that the photodiode is not saturated by the optical carrier which does not contribute to the signal. To maximize the conversion gain an EDFA is used at the output stage of the MZM.

Although these systems allow for very flexible and wideband frequency-converters/transmitters, the implementation using discrete modulators severely limits the scalability and cost-efficiency of this solution. An interesting approach is to use IMWP to implement a similar architecture, but on a silicon photonic platform. A fully integrated photonic frequency down-converter was demonstrated using a custom InP platform [12]. However, this system was developed for RF radar front-ends up to $1 \mathrm{GHz}$ and not applied to wireless communication systems. Currently it is still quite challenging to provide high frequency modulators in compact Photonic Integrated Circuits (PICs). Although carrier-depletion based modulators can provide bandwidths up to $40 \mathrm{GHz}$, the needed voltage swing to drive them remains rather large [13]. A very appealing alternative to carrier-depletion MZMs, are GeSi Electro Absorption Modulators (EAMs). They provide bandwidths exceeding $65 \mathrm{GHz}$, covering a substantial part of the mmWave spectrum. Furthermore, they can be fabricated on a wafer-scale in a $(200 \mathrm{~mm}$ or $300 \mathrm{~mm}$ wafer) silicon photonic pilot line. Radio-over-Fiber links using EAMs have been investigated [14], as well as EAM ARoF with photonic up-conversion [15]. However these results were demonstrated with discrete InP EAMs and the up-conversion was realized using bulky circulators and other discrete components. Not only do the GeSi EAMs have the advantage of wafer-scale fabrication, they can easily be integrated in a PIC with lowloss and compact waveguides, splitters, MZIs and thermo-optic phase-shifters.

In this work we present an ARoF up-converter/transmitter implemented in a silicon PIC. The remainder of this text consists of three parts, in the first part we give a detailed discussion of the silicon photonic up-converter/transmitter implementation and the proposed down-link architecture. In the second part we present the results of the GeSi EAM bandwidth measurement, the up-converter/transmitter linearity measurement and the $2 \mathrm{~km}$ fiber transmission experiment. In the final part we present a conclusion.

\section{DESIGN AND IMPLEMENTATION}

In Fig. 1 the cross section of the EAM waveguide, a schematic overview of the EAM-based mixer-transmitter link 


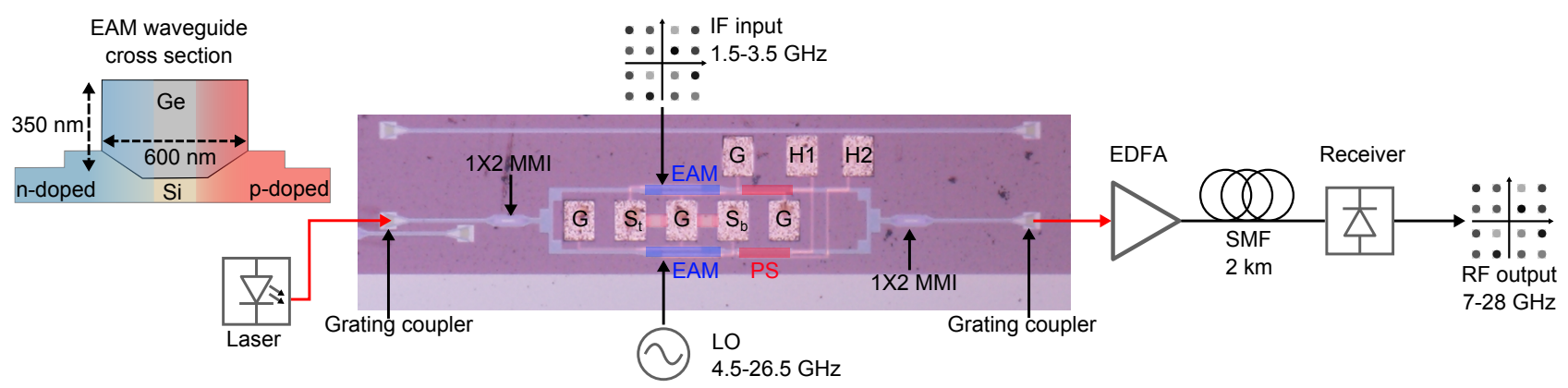

Fig. 1. Cross section of the EAM waveguide and overview of the full up-converter/transmitter link. EAM: Electro Absorption Modulator; MMI: Multi-Mode Interferometer; PS: Phase-Shifter; EDFA: Erbium Doped Fiber Amplifier; SMF: Single Mode Fiber

and a microscope image of the silicon PIC are shown. The key components of the PIC are two GeSi EAMs placed in a MZI structure. The MZI structure is created using passive silicon waveguides and 1 by 2 MMIs. One EAM is placed in the top MZI arm and is used to imprint the data (at IF frequency) on the optical carrier. The other EAM is placed in the bottom arm and is driven by the LO signal needed to up-convert the data to the wanted output RF frequency. The EAMs are connected with metal interconnects to an array of bondpads in G-S-G-SG configuration with a $100 \mu \mathrm{m}$ pitch (as shown in Fig. 1). An on-chip $70 \mathrm{Ohm}$ resistor was included (in between $\mathrm{G}$ and $\mathrm{S}$ pads) to provide broadband matching, resulting in a return loss better than $10 \mathrm{~dB}$ from $10 \mathrm{MHz}$ to $67 \mathrm{GHz}$ (see Fig. 2). For narrow-band operation, a dedicated matching network could be used which would improve the conversion gain. The PIC contains two grating couplers which allow the PIC to be interfaced with cleaved single mode fiber (SMF). The MZI structure also contains a thermo-optic phase-shifter which is used to apply a static $\pi$ phase-shift to the MZI structure which suppresses the optical carrier. This is important, because in this microwave photonic mixer architecture the optical carrier does not contribute to the output signal but can saturate the amplifier and receiver. Furthermore, by suppressing the carrier, the LO leakage at the receiver is also mitigated. This architecture closely resembles previously demonstrated photonic frequency-converters with $\mathrm{LiNbO} 3 \mathrm{MZMs}$ in each arm of a MZI structure [10]. In this implementation however, the amplitude modulators (MZMs) are replaced by intensity modulators (EAMs). As shown in Fig. 1 the envisioned downlink consists of a laser, the PIC, standard SMF, an optical amplifier and a PD. In this work the link was demonstrated with an external laser, but a fully integrated version of the PIC could be realized by the heterogeneous integration of a DFB laser onto the silicon PIC [16]. Similarly, the amplification stage at the PIC output was implemented with an EDFA in this work. However, depending on the system requirements a SOA could be heterogeneously integrated on the PIC [17]. Also on the receiver side a commercial device was used, however as we have demonstrated in previous work a silicon PIC with GeSi photodiodes co-integrated with a TIA could also be used as receiver [18]. The GeSi EAMs have a bandwidth exceeding $65 \mathrm{GHz}$ and therefore both the IF input and LO frequency can be extended to $60-70 \mathrm{GHz}$ bands. This implies that the

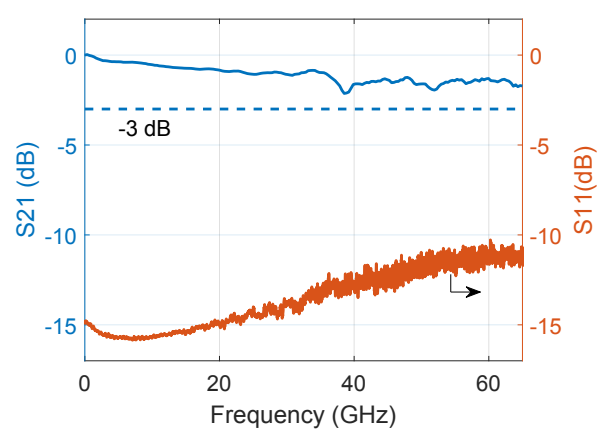

Fig. 2. Small-signal frequency response and return loss of the GeSi EAM measured with a Keysight PNA-X vector network analyzer.

same structure can also be used for microwave photonic upconversion and down-conversion of mmWave bands without any modification of the PIC. The carrier frequencies shown in Fig. 1 were used in the transmission experiment described in the following section and were limited by measurement equipment.

The EAMs have an extremely small footprint of 20 by $80 \mu \mathrm{m}$, which is an order of magnitude smaller than integrated silicon photonic MZMs. This implies that a large number of these mixers can be integrated on one PIC for wavelength multiplexing or multi-core fiber interfacing, as was shown for $896 \mathrm{~Gb} / \mathrm{s}$ digital data transmission [19].

\section{MeAsurement Results}

Before characterizing the up-conversion/transmitter we first carried out a small-signal measurement of a single EAM to determine the bandwidth and ripple. For this purpose we used a PNA-X vector network analyzer together with a $\mathrm{u}^{2} \mathrm{t}$ $50 \mathrm{GHz}$ XPDV2320R photodiode. To accurately determine the small-signal response up to $65 \mathrm{GHz}$, the frequency response of the photodiode was de-embedded. A measurement of the frequency depended response of a GeSi EAM shows less than $1 \mathrm{~dB}$ ripple and a $3 \mathrm{~dB}$ bandwidth beyond $65 \mathrm{GHz}$. The result of this measurement is shown in Fig. 2.

To characterize the GeSi EAM up-convertor/transmitter, the PIC was placed on a temperature controlled chuck and kept at $20^{\circ} \mathrm{C}$. The optical input- and output were provided using cleaved SMF. The layout of the measurement set-up is shown 


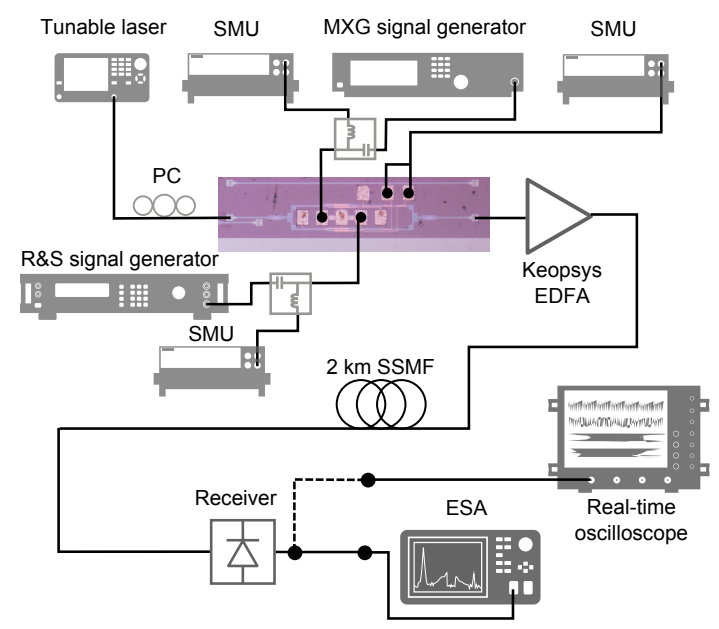

Fig. 3. Layout of the measurement set-up for the $10 \mathrm{MBd}$ 64-QAM signal over $2 \mathrm{~km}$ of standard SMF transmission experiment. SMU: Source Measure Unit; PC: Polarization Controller; SSMF: Standard Single Mode Fiber; EDFA: Erbium Doped Fiber Amplifier, ESA: Electrical Spectrum Analyzer.

in Fig. 3. A tunable laser is used with an output power of 10 $\mathrm{dBm}$ at a wavelength of $1565 \mathrm{~nm}$. The EAMs were contacted using a G-S-G-S-G RF probe with $100 \mu \mathrm{m}$ pitch. Each EAM was reverse biased with $0.8 \mathrm{~V}$ and the heater was used to bias the MZI structure at minimum transmission point. The dynamic insertion loss of the EAM at a reverse bias of $0.8 \mathrm{~V}$ is approximately $8 \mathrm{~dB}$. The LO EAM was driven with a Rhode and Schwarz signal generator (RS SG). This EAM could be driven with high power $(10 \mathrm{dBm})$ without danger of compromising the linearity as the generated harmonics are out-of-band.

To determine the linearity of the mixer, the IF EAM was driven with a Keysight MXG signal generator (MXG SG). The MXG SG was used to generate two-tone signal for an IP3 linearity measurement, with the tones centered at $1.5 \mathrm{GHz}$ and spaced by $0.2 \mathrm{MHz}$. The IF input power was varied while the LO power was kept constant at $10 \mathrm{dBm}$.

To determine the inter-modulation distortion and the resulting up-converted signal, the signal power at $26000.1 \mathrm{MHz}$ and the inter-modulation power at $26000.3 \mathrm{MHz}$ were measured. The result of the measurement is shown in Fig. 4. The measured Spurious Free Dynamic Range (SFDR) exceeds $82 \mathrm{~dB} \cdot \mathrm{Hz}^{2 / 3}$, and the input third order intercept point (IIP3) occurs at $25 \mathrm{dBm}$ of IF input power. The SFDR is comparable to earlier work on EAM based RoF links [14][20] and could be further improved by lowering the noise floor.

To characterize the photonic system as transmitter with photonic up-conversion, we performed a transmission experiment over $2 \mathrm{~km}$ of SMF as described in Fig. 3. In a first transmission experiment a $10 \mathrm{MBd} 64-\mathrm{QAM}$ signal was generated on a $1.5 \mathrm{GHz}$ IF carrier using the MXG SG. At the output of the PIC a Keopsys EDFA (CEFA-HG) is used to amplify the optical signal power to approximately $-4 \mathrm{dBm}$. Although in mmWave frequency bands higher baud rates are of interest, in this experiment we were limited by the modulation bandwidth of the MXG SG. The 64-QAM signal was used to drive the IF EAM, while the LO EAM was driven using the R\&S SG with

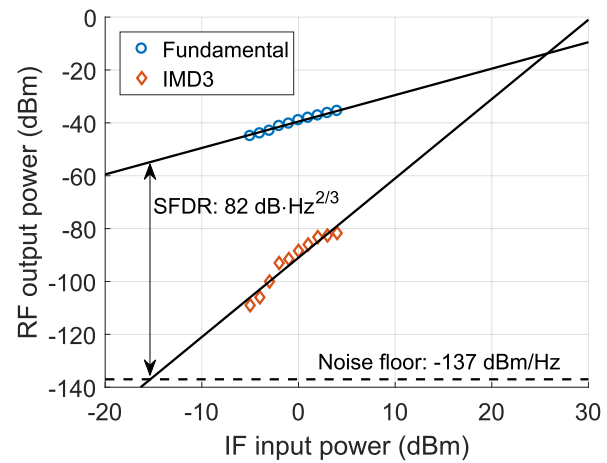

Fig. 4. Results of the linearity (IP3) measurement as function of the IF input power, with a fixed LO power of $10 \mathrm{dBm}$. The output power was measured at the photodiode output stage. SFDR: Spurious Free Dynamic Range; IMD3: third-order Inter-Modulation Distortion.

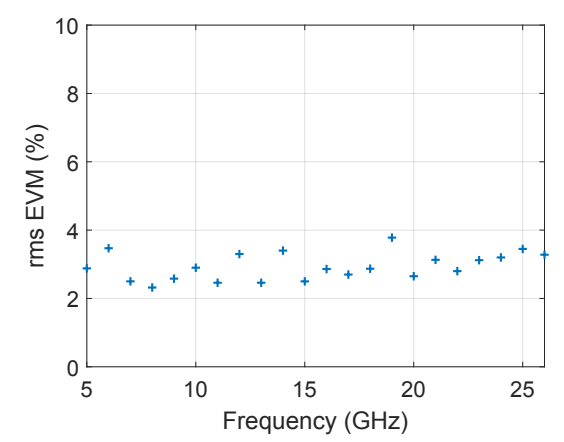

Fig. 5. The rms EVM of a received $10 \mathrm{Mbd}$ 64-QAM signal as function of the received carrier frequency. The IF had a frequency of $1.5 \mathrm{GHz}$ and an input power of $0 \mathrm{dBm}$, and $\mathrm{LO}$ input power of $10 \mathrm{dBm}$.

a frequency varying from 4.5 to $24.5 \mathrm{GHz}(10 \mathrm{dBm})$. In this experiment a linear PIN-TIA optical receiver (Discovery DSC409) was used and the output of the receiver was analyzed using a Keysight MXA electrical signal analyzer (MXA SA). The LO frequencies were chosen to up-convert the IF signal to the $>6 \mathrm{GHz}$ bands, while the maximum RF carrier frequency was limited by the MXA SA. As shown in Fig. 2 the EAM bandwidth allows up-conversion to carrier frequencies larger than $65 \mathrm{GHz}$ with less than $3 \mathrm{~dB}$ penalty. The rms Error Vector Magnitude (EVM) of the received 64-QAM signal was determined online using the native software of the MXA SA, without the use of equalization. The rms EVM was normalized to the maximum amplitude of the constellation. The rms EVM of 1000 symbols for the 64-QAM $10 \mathrm{MBd}$ signal as function of the received carrier frequency is shown in Fig. 5. Although the specifications for $5 \mathrm{G}$ RANs are not defined, the 3GPP defined a maximum rms EVM for current LTE RANs of $8 \%$ for transmitted 64-QAM signals and $3.5 \%$ for 256-QAM signals.

To investigate the transmission of higher baud rates, an in-house programmed FPGA signal generator was used to create a $218 \mathrm{MBd}$ 64-QAM signal on a $3.5 \mathrm{GHz}$ carrier. Because the MXA SA is limited to $25 \mathrm{MBd}$ signals, we used a Keysight real-time oscilloscope (DSA-Z 634A) to analyze the received signal. Again, native software on the oscilloscope was used to determine the online rms EVM of the received 


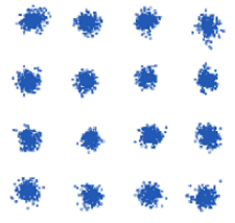

(a)

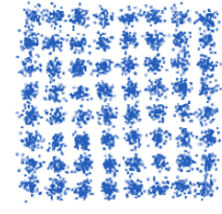

(b)
Fig. 6. (a) Constellation diagram of a received $218.75 \mathrm{MBd}$ 16-QAM data on a $28 \mathrm{GHz}$ carrier with an rms EVM of $5 \%$. (b) Constellation diagram of a received $218.75 \mathrm{MBd}$ 64-QAM data on a $28 \mathrm{GHz}$ carrier with an rms EVM of $5.4 \%$.

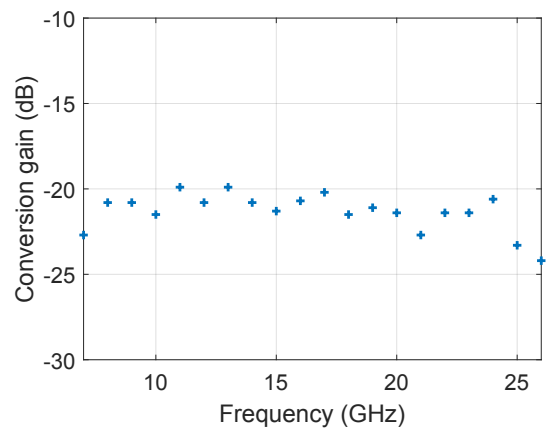

Fig. 7. Conversion gain as function of the carrier output frequency for a LO input power of $10 \mathrm{dBm}$.

signal. This time the RF carrier frequency was limited due to the oscilloscope to $28 \mathrm{GHz}$, which corresponded to a $\mathrm{LO}$ frequency of $24.5 \mathrm{GHz}$. A constellation diagram of both a 16QAM and a 64-QAM received signal, transmitted over $2 \mathrm{~km}$ of SMF, are shown in Fig. 6. The rms EVM of the received data was $5.4 \%$ for $1.3125 \mathrm{~Gb} / \mathrm{s}$ 64-QAM data on a $28 \mathrm{GHz}$ carrier.

We then investigated the conversion gain as function of the LO frequency when an IF tone without data is used. The IF EAM was driven with a $0 \mathrm{dBm}$ and $1.5 \mathrm{GHz}$ tone, while the LO frequency was swept between 4.5 an $24.5 \mathrm{GHz}$ with a constant power of $10 \mathrm{dBm}$. The result of the measurement is shown in Fig. 7. The ripple of the conversion gain is attributed to the probe contact and the cables, as the transmitter was not de-embedded and the response of the EAM should have less than $1 \mathrm{~dB}$ ripple according to Fig. 2.

\section{CONCLUSIONS}

In summary, we demonstrated a microwave photonic upconvertor/transmitter implemented in silicon photonics. We showed that the EAM has a $3 \mathrm{~dB}$ bandwidth exceeding $65 \mathrm{GHz}$ and a ripple smaller than $1 \mathrm{~dB}$. Furthermore, we demonstrated the up-conversion of a $1.5 \mathrm{GHz}$ IF to a frequency range of 7 to $26 \mathrm{GHz}$ with less than $2 \% \mathrm{rms}$ EVM variation over the entire frequency range. This was done for a 64-QAM $10 \mathrm{MBd}$ signal, where both the maximum carrier frequency and baud rate were limited by the measurement equipment. We then also demonstrated the transmission and up-conversion of a $1.3 \mathrm{~Gb} / \mathrm{s}$ 64-QAM signal. Furthermore, in future PICs the external laser and amplifier could be heterogeneously integrated. In this work we presented the system as a transmitter and up-converter, but the PIC could also be used as a photonic microwave down-converter. Finally, due to the very compact footprint of the EAM many of these up-converter/transmitters can be combined and multiplexed on a single chip.

\section{REFERENCES}

[1] W. Zhang and J. Yao, "On-chip silicon photonic integrated frequencytunable bandpass microwave photonic filter," Opt. Letters, vol. 43, no. 15 , pp. 3622-3625, 2018

[2] W. Zhang and J. Yao, "Silicon Photonic Integrated Optoelectronic Oscillator for Frequency-Tunable Microwave Generation," J. of Lightw. Technol., vol. 36, no. 19, pp. 4655-4663, 2018.

[3] D. Perez et al., "Multipurpose silicon photonics signal processor core," Nature Commun., vol. 8, no. 1, pp. 636, 2017

[4] W. Zhang and J. Yao, "A fully reconfigurable waveguide Bragg grating for programmable photonic signal processing," Nature Commun., vol. 9, no. 1 , pp. 1396,2018

[5] Y. Xie et al., "Programmable optical processor chips: Towards photonic RF filters with DSP-level flexibility and MHz-band selectivity," Nanophotonics, vol. 7, no. 2, pp. 421454, 2017.

[6] L. Zhuang, C. G. H. Roeloffzen, M. Hoekman, K. J. Boller, and A. J. Lowery, "Programmable photonic signal processor chip for radiofrequency applications," Optica, vol. 2, no. 10, pp. 854859, 2015.

[7] J. Lota, S. Sun, T. S. Rappaport and A. Demosthenous, "5G Uniform Linear Arrays With Beamforming and Spatial Multiplexing at 28, 37, 64, and $71 \mathrm{GHz}$ for Outdoor Urban Communication: A Two-Level Approach," IEEE Transactions on Vehicular Technology, vol. 66, no. 11, pp. 9972-9985, 2017.

[8] S. Ek et al., "A 28-nm FD-SOI 115-fs Jitter PLL-Based LO System for 2430-GHz Sliding-IF 5G Transceivers," IEEE Journal of Solid-State Circuits, vol. 53, no. 7, pp. 1988-2000, 2018.

[9] C. Ranaweera, E. Wong, A. Nirmalathas, C. Jayasundara and C. Lim, "5G C-RAN With Optical Fronthaul: An Analysis From a Deployment Perspective," J. of Lightw. Technol., vol. 36, no. 11, pp. 2059-2068, 2018.

[10] E. H. W. Chan and R. A. Minasian, "Microwave Photonic Downconverter With High Conversion Efficiency," J. of Lightw. Technol., vol. 30, no. 23 , pp. 3580-3585, 2012.

[11] T. Jiang, R. Wu, S. Yu, D. Wang, and W. Gu, "Microwave photonic phase-tunable mixer," Opt. Express, vol. 25, no. 4, pp. 45194527, 2017

[12] S. Jin, L. Xu, V. Rosborough, J. Klamkin and Y. Li, "RF Frequency Mixer Photonic Integrated Circuit," IEEE Photon. Technol. Lett., vol. 28, no. 16, pp. 1771-1773, 2016.

[13] D. Patel et al., "Design, analysis, and transmission system performance of a $41 \mathrm{GHz}$ silicon photonic modulator,' Opt. Express, vol. 23, no. 11, pp. 1426314287,2015

[14] B. Hraimel et al., "Experimental Demonstration of Mixed-Polarization to Linearize Electro-Absorption Modulators in Radio-Over-Fiber Links," IEEE Photon. Technol. Lett., vol. 23, no. 4, pp. 230-232, 2011.

[15] C. Park, C. Oh, C. Lee, D. Kim, C. Park, "A photonic up-converter for a WDM radio-over-fiber system using cross-absorption modulation in an EAM," IEEE Photon. Technol. Lett., vol. 17, no. 9, pp. 1950-1952, 2005.

[16] J. Zhang, B. Haq, J. O'Callaghan, A. Gocalinska, E. Pelucchi, A. Jose Trindade, B. Corbett, G. Morthier, G. Roelkens, "Transfer-printing-based integration of a III-V-on-silicon distributed feedback laser," Opt. Express, vol. 26 , no. 7 , pp. 8821-8830, 2018.

[17] M. L. Davenport, S. Skendzic, N. Volet, J. C. Hulme, M. J. R. Heck and J. E. Bowers, "Heterogeneous Silicon/IIIV Semiconductor Optical Amplifiers," IEEE J. Select. Topics Quantum Electron., vol. 22, no. 6, pp. 78-88, 2016.

[18] K. Van Gasse et al., "III-V-on-Silicon Photonic Transceivers for RadioOver-Fiber Links," J. of Lightw. Technol., vol. 36, no. 19, pp. 4438-4444, 2018.

[19] P. De Heyn et al., "Ultra-dense 1656Gb/s NRZ GeSi EAM-PD arrays coupled to multicore fiber for short-reach $896 \mathrm{~Gb} / \mathrm{s}$ optical links," 2017 Optical Fiber Communications Conference and Exhibition (OFC), Los Angeles, CA, 2017, pp. 1-3.

[20] K. K. Loi et al., "Low-loss 1.3-m MQW electroabsorption modulators for high-linearity analog optical links," IEEE Photon. Technol. Lett., vol. 10, no. 11, pp. 1572-1574, 1998. 